\title{
The sexual difference around hetero-sexism and queerness; philosophical-sociological perspectives
}

\section{[La diferencia sexual a la vuelta del hetero-sexismo y la queerness; perspectivas filosofico-sociologicas]}

\author{
María J. Binetti - Miroslav Tvrdon - Kristina Klasnja - Tatiana A. \\ Baklashova - Slavka Krasna
}

DOI: 10.18355/XL.2021.14.03.16

\begin{abstract}
The following pages are intended to show why sexual difference constitutes a category that surpasses both the traditional hetero-normative model and the postmodern multiplication of infinite self-determined genders. Both false alternatives are founded on a dualistic framework that separates and excludes some kind of fixed and eternal abstract entities, on the one hand, and on the other, finite signifiers that are increasingly fragmented. Sexual difference, on the contrary, allows us to posit the self-differing and immanent multiplicity of each sex without the need to reify or eliminate them.
\end{abstract}

Key words: resilience, resonance, theological virtues, Hartmut Rosa, our relationship to the world

\section{Resumen}

Las siguientes páginas se proponen mostrar por qué la diferencia sexual constituye una categoría superadora tanto del tradicional modelo hetero-normativo como de la postmoderna multiplicación de infinitos géneros auto-determinados. Ambas falsas alternativas se fundan en un esquema dualista que separa y excluye entidades abstractas supuestamente fijas y eternas, por un lado y por el otro, significantes finitos cada vez más fragmentados. La diferencia sexual, por el contrario, permite afirmar el auto-diferir y la multiplicidad inmanente de cada sexo sin necesidad de cosificarnos ni eliminarlos.

Palabras clave: Fluidez, dialéctica, no-todo, mediación, nomadismo

\section{Introducción}

Las siguientes páginas se proponen inscribir la diferencia sexual femenina como categoría que supera tanto el dualismo hetero-sexista propio del patriarcado más tradicional, como la volatización de incontables identidades de género operada por la ideología queer. En el caso del dualismo hetero-sexista, la identidad femenina es forzada al trágico destino esencialista de una cosa-en-sí, trascendente e inmutable, opuesta al estereotipo masculino. En el caso de las incontables identificaciones de género propias de la ideología queer, se elimina la consistencia ontológica de ser mujer, como si esta fuera un mero producto cultural, lingüístico o económico. A pesar de la aparente distancia entre ambas opciones, ellas coinciden en ser los términos opuestos de un falsa alternativa esencialista vs. anti-esencialista, donde en cualquier caso lo eliminado es la mujer como realidad múltiple, compleja y dinámica, universal en su misma singularidad y concreción. Desde el punto de vista esencialista, la realidad femenina se ubica en una abstracción trascedente, inmutable y eterna; desde el punto de vista anti-esencialista, en un significante vacío capaz de significar atribuible indistintamente a varones o mujeres. En una palabra, heterosexismo compulsivo o incontables identidades de género delimitan la falsa alternativa de un mismo dualismo falogocéntrico y misógino.

XLinguae, Volume 14 Issue 3, June 2021, ISSN 1337-8384, eISSN 2453-711X 
De la mano del fantasma (anti-)esencialista, el post-feminismo gay y queer ha llegado a dominar hoy la escena de los géneros como el abanderado de una empresa emancipadora que ha venido a liberar a las mujeres del sueño dogmático de serlo o, eventualmente, del dogmatismo de no serlo. Conceptualmente expresado, el postfeminismo constituye un post-estructuralismo lingüisticista, de ascendencia más o menos marxista. Frente al imaginario del eterno femenino y el inexpugnable masculino, el constructivismo post-feminista sostiene la producción socio-lingüística de la materialidad sexuada, de manera tal que la ancestral dicotomía entre el elemento material pasivo y receptivo, y la forma viril, activa y generadora, se ve ahora reeditada por la nueva versión falo-logos-lingüisticista.

La disolución trans-genérica y trans-sexual operada por el post-feminismo constituye el punto de llegada obligado de los supuestos constructivistas que convirtieron a la categoría de género en absoluto ontológico y principal recurso estratégico de las políticas feministas oficiales. Es necesario distinguir en este punto el uso heurístico de la categoría de género como instrumento analítico de las ciencias sociales, históricas y antropológicas, cuyo aporte es invalorable, de su traspolación y reificación ontológica, tanto dentro del dualismo metafísico asumido por el sistema sexo-género, como en la versión constructivista del género en tanto que absoluto ontológico, performado por las relaciones sociales, lingüísticas, económicas, etc. En este último caso, el constructivismo genérico desemboca en la disolución de toda identidad sexual más que cultural.

\section{Ni sexo ni género, ni post ni trans}

La prevalencia del "género" en sustitución del "sexo" ha sido difundida y promovida por el discurso sociológico norteamericano, dominado en parte por el constructivismo lingüisticista postmoderno, en parte por la sacralidad absoluta de la libertad individual, en parte por el conservadurismo de corte ético-religioso que convirtió lo sexual en un tabú para las mujeres. Rosi Braidotti comenta al respecto que en el contexto de la moral sexual americana, fuertemente atravesada por elementos conservadores y puritanos, uno de los pocos canales que el feminismo encontró para incorporar una agenda sexual fue a través de los colectivos queer, y sus reivindicaciones en materia de libertad sexual (Dolphijn y van der Tuin, 2012: 26). Bajo el impulso del norte hegemónico, el activismo queer logró imponerse en todo el mundo y particularmente en América Latina, impulsado por programas y agencias de las Naciones Unidas, poderosos lobbies corporativo y políticas populistas con una retórica posmoderna y subjetivista ${ }^{1}$.

La hegemonía de la teoría de género y sus desplazamientos hacia el post-genrismo y trans-generismo ha sido muy cuestionada por varias corrientes feministas, entre ellas el feminismo de la diferencia sexual, y los nuevos feminismos materiales y realistas. Las críticas podrían resumirse en el falogocentrismo y misoginia encubierta de la diseminación performativa queer, cuya feminofobia esconde en rigor, bajo una supuesta neutralidad del sujeto discursivo, una nueva masculinización de la cultura hegemónica y una reapropiación de la fuerza sexo-reproductiva de las mujeres promocionada como "trabajo sexual" y "surrogación" solidaria. Autoras como Rosi Braidotti y Elizabeth Grosz ven en la queerness de los géneros un esencialismo reificante de contra-identidades reactivas, cuya posición marginal al sistema en verdad refuerza los estereotipos que dice superar. Otras autoras provenientes del feminismo lésbico radical como Sheila Jeffreys objetan la incompatibilidad de intereses entre transexualismo masculino y feminismo, a la vez que cuestionan el control social de

\footnotetext{
${ }^{1}$ Aludimos aquí a la afirmación de Maurizio Ferraris sobre la convergencia entre el discurso disolvente de la post-modernidad y las políticas populistas (Ferraris, 2013: 42).
} 
los cuerpos así llamados transexuales, la industria médica farmacéutica que los promueve la autodeterminación sexual y el abuso de menores operados y hormonados bajo el rótulo de ser "trans". El hecho de borrar la diferencia sexual entre varones y mujeres, hace el imaginario queer diluya la desigualdad estructural entre ambos, disimule la posición subordinada de las mujeres, y perpetúe la explotación sexoreproductiva de las mujeres, subsidiaria de los intereses de las diversidades sexuales como el alquiler de úteros y la compra de niños: esa vieja historia falogocéntrica de convertir a las mujeres en vasijas. El supuesto neutro sexual que se pretende instalar en el discurso así llamado inclusivo nunca esconde la supremacía masculina.

Tal como lo h a señalado Catherine Malabou (2011: 99), la violencia socio-política ejercida contra las mujeres es el resultado inexorable de la violencia teórica y la desontologización operada por el constructivismo lingüisticista, donde los cuerpos materiales y sexuados se reducen a mero receptáculo vacío y pasivo de la ingeniería queer, constructora de cuerpos y sexualidades tecno-formadas. Mientras tanto las mujeres reales, de carne y hueso, las que pueden parir o abortar, ser penetradas o violadas, las menstruantes, inseminadas o menopaúsicas, las que venden su vagina y su útero por unos pocos pesos, esas mujeres siguen siendo las más pobres, las viejas, las más solas, las más explotadas del sistema de los incontables géneros.

Así están las cosas desde el punto de vista del generismo queer hegemónico, donde ser mujer es un mero significante cultural y pretender otra cosa constituye un abuso del lenguaje, un residuo reaccionario, y una amenaza hetero-sexista y retrógrada. Sin embargo, frente a la disolución del posmoderna de toda la realidad en ficciones discursivas, el alborear del nuevo siglo nos hace testigos de un nuevo escenario especulativo y político. Junto con el agotamiento del paradigma discursivo para el cual el significante construye el significado, asistimos hoy al giro ontológico y neorealista del siglo XXI, con deslizamientos hacia un nuevo materialismo especulativo. En el ámbito de las teorías feministas, el paradigma de la diferencia sexual se ve renovado por los nuevos feminismos materiales, realistas y científicos. Por otra parte, desde el punto de vista político, somos partícipes de una movilización de mujeres tan masiva como jamás la hubo. Movimientos colectivos como "Ni una menos", “\#NoEsNo", "\#MeToo" o "Time's up" expresan la urgencia de un nuevo pacto sexual entre varones y mujeres, más allá de los incontables géneros y trans-géneros imaginados.

Tal es el contexto en el que consideramos imperiosa la necesidad de reconsiderar la diferencia sexual como categoría ontológica, praxis política y proyecto cultural. A eso apuntarán las siguientes páginas, dedicadas a la diferencia sexual como concepto que supera la falsa opción entre un esencialismo de abstracciones inmutables y un antiesencialismo disolvente de toda realidad, o bien entre biología y cultura, universalidad y pluralismo, etc. Nuestra consideración se moverá en el marco de la nueva especulación del siglo XXI y seguiremos los pasos de las grandes teóricas de la diferencia como Luce Irigaray, Rosi Braidotti y Elizabeth Grosz, quienes han concebido la realidad ontológica de la mujer fuera del esquema dualista esencialismo vs. anti-esencialismo, como realidad consistente y a la vez fluida, nomádica y volátil, capaz de reinventarse continuamente a sí misma en el juego auto-determinante de la diferencia sin perder por eso su profunda identidad.

\section{La historia especulativa de la diferencia}

El concepto de "diferencia sexual" elaborado por el feminismo tiene una larga historia filosófica que se remonta al idealismo absoluto y se extiende hasta nuestros días con el nuevo giro especulativo y material del siglo XXI, pasando por la elaboración de la différence o différance del post-estructuralismo francés. El logro especulativo del idealismo, legado por lo demás a la posteridad contemporánea, consistió en superar el dualismo esencialista de la lógica y la metafísica clásicas -reinantes por más de 2500

XLinguae, Volume 14 Issue 3, June 2021, ISSN 1337-8384, eISSN 2453-711X 
años- en virtud del monismo dinámico y dialéctico del sujeto absoluto. Mientras que el modelo de la metafísica clásica se basa en la separación dualista entre la perfección del ser trascendente -eterno, inmutable y auto-subsistente- y la imperfección del ser material -temporal, contingente y relativo a aquella-; el paradigma monista del idealismo se sostiene en el despliegue inmanente, reflexivo y auto-diferenciante de un único ser, dialécticamente tensionado por su propia negatividad esencial.

La nueva especulación moderna se propuso reemplazar los principios de la identidad y no contradicción abstractas de la lógica y metafísica clásicas por el principio de la contradicción absoluta como origen y elemento de lo real, o bien, por el primer principio de una identidad subsistente en su propia diferencia. En palabras de Hegel, la especulación idealista incorpora "la identidad en la diferencia y la diferencia en la identidad" (2005: $§ 118$ ), con el resultado de una realidad en continuo devenir autodiferencial. La dialéctica de la identidad asimila el viejo régimen dualista de perfecciones vs. imperfecciones, eternidades vs. contingencias al desdoblamiento inmanente de una misma realidad, atravesada por su propia negatividad o diferencia. En tal sentido, mientras que la tradición sustancialista expulsó lo negativo y diferente al lugar de opuesto excluyente de una positividad simple e igual, la nueva lógica especulativa identifica su oposición con el desdoblamiento esencial de lo mismo, siempre otro.

El post-estructuralismo francés denomina différence o différance a un tipo de diferencia no dualista y sin contrarios, que es ella misma auto-diferenciante y autodeterminante. La diferencia abordada no se opone a la identidad porque no es en rigor ni identidad ni diferencia, ni presencia ni ausencia, ni ser ni representación, ni interioridad ni exterioridad, ni determinación ni indeterminación, ni posición ni privación, etc. Vale decir, se trata de una diferencia absoluta y radical, cuyo dinamismo auto-diferencial y creador está en el origen de todas las cosas. La diferencia aludida opera por encima de las abstracciones intelectuales en tanto que concepto absoluto, superador de las contradicciones y síntesis dinámica de un "tercer género" (Derrida, 1993: 16) de realidad sintético y medial. La différence no es sustancia sino devenir, tampoco es acto perfecto sino virtualidad pura cuyo despliegue auto-diferencial produce las infinitas diferencias de la finitud.

Con el avance del siglo XXI, la identidad dialéctica del idealismo recala en la última filosofía francesa de autores como Slavoj Žižek y Catherine Malabou, quienes pertenecen en un amplio sentido al nuevo giro realista o especulativo. A diferencia del paradigma post-estructuralista dominado por el giro lingüístico, la hermenéutica y el constructivismo cultural, la última ontología francesa reivindica el acceso a lo real en su consistencia irreductible, que justamente no consiste en la simplicidad irreflexiva del realismo sustancialista, sino en el desdoblamiento diferencial de la realidad especulativa. La diferencia, lo negativo, la contradicción esencial determinan el dinamismo de una identidad dialéctica en permanente ruptura, desajuste y tensión consigo misma, así como en continua regeneración. En los términos de Žižek, la diferencia se inscribe como no-Todo (Zizek, 2014: 1; Bryant - Srnicek - Harman, 2011: 407) de una realidad siempre incompleta, desajustada y abierta a la novedad del devenir.

La línea especulativa aquí trazada - con el apuro impuesto por la brevedad de estas páginas- constituye el marco teórico del feminismo de la diferencia, el cual intenta elaborar un concepto de la diferencia que supere el dualismo propio del principio de identidad y no contradicción. El feminismo no inventa, sino que recoge un concepto de diferencia largamente elaborado por la filosofía moderna y contemporánea, a la vez que se afirma sobre la superación del paradigma dualista. Sin embargo, tampoco se trata de una mera recepción histórica, sino de una asimilación transformadora, donde la concepción de la diferencia será reinterpretada a partir de sexualidad como origen y sentido concreto del ser. El hecho de que la diferencia asumida presuponga la consistencia especulativa de la misma, previene el común error de recaer en una 
lectura dualista o sustancialista de la diferencia sexual. Asimismo, el hecho de que se trate de una concepción de la diferencia ontológica basada en el sexualidad femenina como lugar del origen, vale decir, en el cuerpo de la mujer, anticipa la paradigma cultural hegemónico, basado justamente en el falo masculino.

El feminismo conceptualiza la identidad femenina en términos de diferencia ontológica y, por acción recíproca, reelabora la diferencia ontológica a partir de la identidad femenina como lugar del origen, sentido y estructura del ser. La reciprocidad de ambas determinaciones resulta en una nueva ontología y cultura feminista, sexualizadas y realizadas conforme con su origen materno-material. Si la identidad sustancialista del dualismo abstracto sigue el modelo masculino de un ser arrojado fuera de la nada y fuera de la causa trascendente, la identidad dialéctica de la feminista sigue el modelo de la inmanencia material y matricial, vale decir, de un ser concebido, gestado y nutrido por el desdoblamiento matricial del origen. $\mathrm{Su}$ diferenciación intrínseca constituye la condición de posibilidad para una transformación radical del pensamiento y la política.

En lo que sigue, intentaremos precisar el concepto de diferencia sexual tal como lo elabora la ontología feminista.

\section{La diferencia ontológica es diferencia sexual}

La diferencia sexual es diferencia ontológica y, en términos feministas, es la diferencia esencial y absoluta interpretada a partir de la identidad femenina como lugar de origen y nacimiento. Desde el punto de vista ontológico, la diferencia expresa el desdoblamiento reflexivo, negativo y contradictorio de la identidad, vale decir, el auto-diferir creador y determinante de la identidad. Para ello, la diferencia debe contener un margen de indeterminación a partir del cual transformarse. En palabras de Elizabeth Grosz, "el estatus ontológico de la diferencia sexual implica una indeterminación fundamental capaz de explicar la apertura, la incompletitud y las posibilidades de ser completado, suplementado por un reordenamiento posterior" (1995: 80). La negatividad indeterminada de la diferencia contiene virtualmente la posibilidad infinita de su devenir, capaz de liberar la identidad a su propio poder transformador. A lo largo del devenir, la instancia negativa o diferencial se mantiene siempre, dispuesta en tensión dialéctica con cada una de sus determinaciones particulares y contingentes. Cualquier determinación particular conserva la indeterminación de su origen y de ese modo mantiene siempre abierta la posibilidad de una nueva creación. Tal es la consistencia ontológica de la diferencia sexual, cuya negatividad, incompletitud y apertura esencial son potencia de generación y nacimiento.

Como instancia ontológica originaria y radical, la diferencia sexual es un punto de partida inmediato, un factum irreductible, material e histórico, presupuesto a toda otra ulterior determinación. Sin embargo, precisamente por su dinamismo reflexivo, su inmediatez es resultado de un desdoblamiento interior que la tensiona y mediatiza. Cuando autoras como Luce Irigaray o Luisa Muraro aluden a la inmediatez irreductible de la diferencia sexual, lo significado es justamente esa mediación inmanente por el cual la identidad se concibe en su propia determinación. El factum de su inmediatez resulta siempre mediado por múltiples elementos que integran su realidad dinámica y sintética. A saber, lo físico y lo síquico, lo natural y lo cultural, lo material y espiritual, lo pre-lingüístico y lo lingüístico, etc. Luisa Muraro afirma al respecto que la diferencia se presenta siempre "revestida, travestida, traducida y sobre-interpretada" (Diotima, 1995: 112) por diversos flujos dinámicos, de manera que no subsiste sustancialmente en sí misma, sino activamente a través una dialéctica auto-diferencial.

La diferencia sexual opera por fuera de la dualidad entre sexo biológico y género cultural, como presupuesto indeterminado de toda diferenciación y determinante ella

XLinguae, Volume 14 Issue 3, June 2021, ISSN 1337-8384, eISSN 2453-711X 
misma de su devenir subjetivo. Ella no es ni mera construcción cultural ni pura inmediatez natural, porque no hay nada que sea pura construcción cultural o mera inmediatez dada. Por el contrario, toda realidad media naturaleza y cultura, cuerpo y pensamiento, materia y mente. Respecto de las oposiciones binarias, la diferencia sexual opera como tercer-género medial, reflexionando, diferenciando y relacionando los elementos de su identidad sintética, de manera tal que el dualismo es superado en y por su mediación. Naturaleza y cultura, materia y espíritu, sustrato dado y construcción histórica, necesidad y contingencia, intimidad subjetiva y exterioridad física, etc. resultan -en y por la mediación dialéctica de la diferenciadeterminaciones abstractas de una misma identidad relacional. Lia Cigarini subraya en este sentido la eficacia productora de la diferencia sexual como "mediación universal" (1996: 229), apuntando con ello a la capacidad de concebirse en su propia alteridad.

A la consistencia negativa y medial de la diferencia se suma su materialidad esencial, síntesis de múltiples elementos y energías corporales en permanente transformación. La diferencia sexual emerge con el cuerpo sexuado como núcleo de su materialidad y permanece -comenta Rosi Braidotti- "codificada en la carne como una memoria elemental, como un banco de datos genéticos que precede a la entrada en la representación simbólica" (2005: 67). El sujeto de la diferencia sexual es carne, cuerpo y encarnadura que marca todo devenir existencial por su actualidad irrevocable. Ahora bien, justamente la inmanencia activa de la diferencia en el corazón de la corporalidad sexuada reconfigura los propios conceptos de materia y sexo liberándolos del determinismo biologicista que el sesgo constructivista atribuye a cualquier consideración biológica. En concreto, la materia implicada por la diferencia sexual no es la clásica materialidad del dualismo intelectualista: mero receptáculo pasivo de formas activas, espirituales, culturales o lingüísticas. Se trata antes bien de una materialidad autodinámica y diferencial, productora ella misma -por su propia diferencia inmanente- de su devenir material y existencial. En el mismo sentido, el sexo del cual se habla no es el mero receptáculo de configuraciones culturales o relaciones sociales, ni tampoco el rígido destino del esencialismo. El sexo de la diferencia constituye antes bien, siguiendo el análisis de Sarah S. Richardson (2013), un dinamismo diferenciante, configurado de manera diádica y relacional. Este tipo de materialidad sexuada es la que sostiene tanto los feminismos de la diferencia como los nuevos feminismos materiales y ontológicos.

En esta misma línea argumental, podemos afirmar que la diferencia sexual es ella misma una realidad esencial, sin que esto signifique una recaída en el esencialismo o en la vacua discusión dualista esencialismo vs. anti-esencialismo, como si la única concepción ontológica de la esencia fuese la de una entidad eterna, inmutable y trascendente que define de manera necesaria el devenir de lo inesencial. Por el contrario, en la línea especulativa que venimos siguiendo, la esencia expresa el dinamismo reflexivo y relacional de la identidad, su negatividad inmanente en virtud de la cual ella se libera como energía auto-determinante (cf. Hegel, 2005: § 112). La consistencia negativa y transformadora de la esencia ha sido subrayada por Catherine Malabou, para quien la feminidad constituye el lugar de continuas transformaciones (2011: 136). Y otro tanto puede decirse de la subjetividad femenina como conciencia concreta, material, dinámica y siempre situada, en contraste con la conciencia clara y distinta del intelectualismo sustancialista.

La diferencia sexual no se alcanza sumando o restando particularidades empíricas y contingencias socio-culturales, porque ella no es la generalización estadística del universal abstracto. A ella tampoco se llega por comparaciones extrínsecas del binomio masculino/femenino, como si la diferencia se jugara en la oposición relativa de dos términos excluyentes. De la diferencia sexual se parte como lugar de origen, inmediatez irreductible que es siempre indeterminación, mediación y autodeterminación libre. Por eso ella no se mide en términos dualistas ni con la identidad ni con la diferencia masculina, sino en términos relacionales con su propia 
potencialidad creadora. Su negatividad interior constituye el sustrato de una continua auto-determinación que es verdad, estrictamente hablando, su libertad creadora.

Lejos de dividir el mundo en dos mitades opuestas que se excluyen mutuamente -la femenina y la masculina- el concepto de diferencia sexual significa el desdoblamiento inmanente de la identidad en su propio diferir realizador y transformador. Autores como Rick Dolphijn y Iris van der Tuin hablan en este sentido de sexual differing (2012: 141 ss.) a los efectos de subrayar la consistencia dinámica y autopoiética de la identidad, emergente de su propia virtualidad material.

En una palabra, la diferencia sexual no se alcanza por afuera de ella misma. De ella se parte como absoluto ontológico del cual no es posible abstraer y en el cual es posible concebir, gestar, dar a luz, regenerar.

\section{Algunas pensadoras de la diferencia}

A título ilustrativo, quisiéramos repasar brevemente la conceptualización de algunas de las grandes teóricas del pensamiento de la diferencia sexual como son Luce Irigaray, Rosi Braidotti y Elizabeth Grosz, las cuales coinciden en afirmar la diferencia en sí y por sí de la identidad femenina, fuera del dualismo hetero-sexista, los estereotipos de género y el constructivismo socio-discursivo. En líneas generales, podríamos decir que las tres comparten una ontología de tipo material y dinámica, donde la diferencia sexual se pone como origen diferencial continuamente desplazado a su alteridad. De la diferencia nace y se nutre un dinamismo material que determina la identidad femenina en términos de "fluidez" para Irigaray, "nomadismo" para Braidotti, y "volatilidad" según Grosz.

Bajo el concepto de "fluidez" (Irigaray, 1985: 107 ss.; Stephens, 2014: 186-202) Irigaray describe una esencia femenina que no es -dualistamente- ni uno ni otro, sino una unidad múltiple, dinámica, indeterminada y auto-diferencial. La idea de lo fluido y su mecánica propia, que la autora opone a la mecánica de lo sólido, evocan intuitivamente los elementos primarios de la vida: sangre, agua, aire, etc., ancestralmente elaborados por la simbólica hilozoísta de la conciencia primitiva como arché material de todas las cosas. Lo fluido acusa la incorporación de la diferencia: asume su intensidad transformadora, resuelve sus tensiones y subsiste en su devenir. La fluidez irigariana es la metonimia de una identidad en permanente cambio y repetición, no sólidamente igual, sino "indefinidamente otra en sí misma" (Irigaray, 1985: 28) y "siempre varias" (Irigaray, 1985: 31). Este modus essendi de lo femenino expresa para Irigaray la actualidad inmanente del origen material-materno-matricial, cuya virtualidad infinita asegura la posibilidad siempre nueva del devenir.

Con declarada ascendencia irigariana, Rosi Braidotti ubica la diferencia sexual en el contexto de una "teoría nomádica" (Bradotti, 1994) que la diferencia misma justifica, contiene y alimenta. Las identidades nómadicas de Braidotti unifican en cada sujeto múltiples procesos diferenciales, a través de los cuales despliegan su virtualidad material, fecunda y creadora. Tal nomadismo auto-diferencial es esencialmente feminista en la medida en que se funda sobre un sustrato materno/material autoactivo, específicamente femenino y ontológicamente universal. En consonancia con Luce Irigaray y Margaret Whitford, Braidotti entiende que "lo material/materno es la instancia que expresa la especificidad de la sexualidad femenina en el sentido de una humanidad femenina y también de su divinidad" (2005: 39-40). Retomando las precisiones hechas líneas atrás, la materia específica de la sexualidad femenina es dinámica, auto-diferencial y creadora, por ser esencialmente negativa, indeterminada y relacional. De su mediación nace lo otro y en su alteridad ella misma se concibe como fuerza de realidad y sentido.

La conclusión de Braidotti es la de una feminidad capaz de concebir, contener y alimentar múltiples y diversos flujos de realización, muchos opuestos y hasta contradictorios. En palabras de la autora, "la cuestión central es el estatus de lo

XLinguae, Volume 14 Issue 3, June 2021, ISSN 1337-8384, eISSN 2453-711X 
femenino material/materno, y las muchas y potencialmente contradictorias maneras en las que continúa afectando la constitución de la subjetividad y las sexualidades femeninas" (2005: 134). Porque la identidad femenina es un diferir activo y actual, es necesario concluir con Braidotti en la posibilidad de múltiples sexualidades femeninas, es decir, de múltiples formas de deseo y goce sexual abiertos a su propia determinación y claramente dispuestos más allá de los estereotipos hetero-sexistas o hetero-genéricos. Sexualidades nomádicas que nacen y se alimentan de la plasticidad materno-material de la identidad femenina.

Cercano a Braidotti se encuentra el feminismo de Elizabeth Grosz, en quien replica igualmente la herencia irigariana. Los "cuerpos volátiles" de Grosz (1994), vibrantes de energía vital, dan cuenta de una materialidad auto-diferenciante y dinámica, en continuo desplazamiento libre y temporal. Para Grosz, la diferencia sexual es "diferencia vital" (1995: 29), porque la vida es siempre sexuada y la sexualidad es creadora de vida. La afirmación de Grosz vale en un doble sentido. Por una parte, ella asume la consistencia auto-diferencial de la diferencia como origen inmanente de lo real. Por la otra parte, la autora adhiere a la premisa irigariana de que los sexos son, por lo menos, dos y "hay una irreductible especificidad de cada sexo relativa al otro" (Grosz, 1995: 77). Vale la pena distinguir en este punto la idea de dualidad sexual femenino/masculina en el sentido en que la piensan Irigaray, Grosz o incluso Richardson, del dualismo hetero-sexista o hetero-genérico, fundado sobre una identidad abstracta y sustancialista. El hecho de que la dualidad sexual constituya un dinamismo relacional, interactivo y creador no niega, sino más bien supone la consistencia auto-activa y auto-diferencial de la diferencia ontológica materno/material, de la cual resulta expresión y medida.

En virtud de su materialidad auto-diferenciante, la sexualidad femenina resulta también para Grosz múltiple, polimorfa, compleja e impredecible. "Cada sexo -afirma la autora en este sentido- tiene la capacidad de jugar con, de devenir diferentes sexualidades, pero no de tomar el cuerpo y sexo de otro" (1995: 77). La multiplicación de sexualidades, en el sentido de explorar nuevas formas de goce y de deseo, viene a confirmar la potencialidad auto-activa de la diferencia sexual, capaz de construir "extrañas carnalidades" (Grosz - Probyn, 1995) móviles, fluidas y siempre abiertas a su libre determinación. En el caso de Grosz o Braidotti, la superación de los estereotipos de género no obedece a la repetición fonética de discursos y significantes -tal como lo piensa el constructivismo posmoderno-, sino al polimorfismo e indeterminación original de la materialidad sexuada, en definitiva tan imprevisible, provisoria y cambiante como la existencia misma. La propuesta de Groz concluye entonces -en lugar de negar la identidad femenina- en volver indecidible y queer la propia sexualidad por la expansión de sus potencialidades materiales.

En esta expansión material, sexual y cultural radica el proyecto político del feminismo de la diferencia, alimentado por la fuerza creadora de la identidad femenina.

\section{A modo de conclusión: la diferencia sexual como praxis y proyecto}

La diferencia sexual no es un problema a resolver ni un obstáculo a eliminar, sino una grandeza a expandir y potenciar. Tal es el punto de partida irreductible del feminismo de la diferencia, cuyos esfuerzos se concentran en superar el sentido dualista y sustancialista de la diferencia, a fin de recuperar su inmanencia originaria y su fuerza de creación.

La identidad femenina que intentamos pensar expresa un modo de ser esencial, material, capaz de concebir, contener y dar nacimiento, cuya diferencia específica se despliega fuera de todo dualismo representativo, en la inmanencia de una mediación superadora. Por eso decíamos que ser mujer no es serlo abstractamente en una única manera posible, sino serlo concretamente en la síntesis única e irrepetible de su universalidad siempre contingente, capaz de decirse en las infinitas maneras posibles de la creación material. 
La acción creadora que ser mujer implica, abraza toda forma de generación material, por lo cual su liberación anticipa una nueva cultura feminista y sexuada en la que reside el gran proyecto de la diferencia sexual como acción política transformadora. El proyecto político de la diferencia es capaz de contener las energías más diversas en la unidad más radical, incluso en medio de tensiones y quiebres internos siempre necesarios al devenir diferencial. En líneas generales, este proyecto podría resumirse en la idea de sexualizar la cultura, no en el sentido dualista de dividir el mundo en dos sexos opuestos, sino en el sentido monista e inmanente de mater/realizar la economía, la ética, el derecho, la religión, etc., por su restitución al origen vivo, deseante y relacional del cual se nace.

En el ámbito específico del ejercicio de las sexualidades, el proyecto de la diferencia nos retrotrae más allá de los estereotipos de género a la intimidad esencial de los cuerpos hecha de ginérgia auto-diferencial. En palabras de Braidotti, él implica "repensar la sexualidad sin los géneros, empezando por un retorno vitalista a la polimórfica, y según Freud perversa, estructura de la sexualidad humana. Necesitamos también revalorizar los poderes generativos del cuerpo femenino" (2013: 98). En el ámbito más amplio de la transformación cultural, la sexualización de la cultura supone su reconcepción en los términos materiales, relacionales y recíprocos de la matriz original. Las autoras de la diferencia proponen en este sentido una cultura del ser-dos en mutuo reconocimiento esencial (Irigaray, 2000: 141-42), lo cual implica el autoreconocimiento en el no-todo, la incompletitud y radical apertura de la diferencia.

En una palabra, el proyecto político de la diferencia sexual se resume en una identidad siempre desdoblada, capaz de contener y alimentar al otro no menos que de ser cobijada y nutrida por la alteridad esencial de la cual es nacida.

\section{Bibliographic references}

BRAIDOTTI, R. 2013. The Posthuman. Cambridge: Polity Press. ISBN 13: 978 0745641584

BRAIDOTTI, R. 2005. Metamorfosis. Hacia una teoría materialista del devenir. Trad. Ana Varela Mateos. Madrid: Akal. ISBN 978-84-460-2067-7

BRAIDOTTI, R. 1994. Nomadic Subjects: Embodiment and Sexual Difference in Contemporary Feminist Theory. New York: Columbia University Press. ISBN 9780231082358

BRYANT, L. - SRNICEK, N. - HARMAN, G. (Eds.). 2011. The Speculative Turn: Continental Materialism and Realism. Melbourne: Re.Press. ISBN 9780980668346

CIGARINI, L. 1996. La política del deseo. La diferencia femenina se hace historia. Trad. Maria-Milagros. Rivera Garretas. Barcelona: Icaria. ISBN 9788474262988

DERRIDA, J. 1993. Khôra. Paris: Galilée. ISBN 2718604239

DIOTIMA. 1995. Oltre l'uguaglianza. Le radici femminili dell'autoritá. Napoli: Liguori Editore. ISBN 9788820724696

DOLPHIJN, R. - VAN DER TUIN, I. 2012. New Materialism: Interviews and Cartographies. Michigan: Open Humanities Press. ISBN 978-1-60785-281-0

FERRARIS, M. 2013. Manifiesto del nuevo realismo. Madrid: Biblioteca Nueva. ISBN 9788499407296 GROSZ, E. 2011. Becoming undone: Darwinian Reflexion on Life, Politics, and Art. Durham \& London: Duke University Press. ISBN 978-0-82235071-2

GROSZ, E. 1995. Space, Time, and Perversion. Essays on the Politics of Bodies. New York: Routledge. ISBN 9781315656564

GROSZ, E. 1994. Volatile Bodies. Toward a Corporeal Feminism. Bloomington \& Indianapolis: Indiana University Press. ISBN 10: 0253208629

GROSZ, E. - PROBYN, E. (Eds.). 1995. Sexy Bodies. The Strange Carnalities of Feminism. New York: Routledge. ISBN 9780203147825

XLinguae, Volume 14 Issue 3, June 2021, ISSN 1337-8384, eISSN 2453-711X 
HEGEL, G. W. F. 2005. Enciclopedia de las ciencias filosóficas en compendio: para uso de sus clases. Trad. R. Valls Plana. M Madrid: Alianza. ISBN 10: 8420681938

IRIGARAY, L. 2000. Democracy begins between two. Trad. Kirsteen Anderson. London: The Athlone Press. ISBN 9780415918176

IRIGARAY, L. 1985. This Sex which is not one. Trad. Catherine Porter y Carolyne Burker. Ithaca: Cornell University Press. ISBN 10: 0801493315

JEFFREYS, S. 2003. Unpacking Queer Politics. A Lesbian Feminist Perspective. Cambridge: Polity Press. ISBN 10: 0745628389

KOBYLAREK, A. 2010. Integration of Elderly Citizens through Learning. In: New Educational Review, vol. 22, n. 3-4, 24 p. ISSN 1732-6729

MALABOU, C. 2011. Changing Difference. The Feminine and the Question of Philosophy. Trad. Carolyn Shread. Cambridge: Polity Press. ISBN 9780745651095

RICHARDSON, S. S. 2013. Sex Itself. The Search for Male \& Female in the Human Genome. Chicago \& London: University of Chicago Press. ISBN 10: 022608468X

RYABCHENKO, O.N. - PROKOPYEV, A.I. - ROMANCHENKO, L.N. KORZHUEV, A.V. - KROKHINA, J.A. 2018. Social and philosophical understanding of national and civic identity in the context of interethnic and interreligious conflict risks. In: XLinguae. vol. 11, n. 2, pp. 359-369, ISSN 2453$711 \mathrm{X}$

STEPHENS, E. 2014. Feminism and New Materialism: the Matter of Fluidity. In: Interalia. A Journal of Queers Studies, vol. 9, pp. 186-202. ISSN 1689-6637

ZIZEK, S. 2014. Absolute Recoil. Towards A New Foundation of Dialectical Materialism. London \& New York: Verso. ISBN 10: 9781781686829

Words: 5631

Characters: 38035 (21,13 standard pages)

Prof. Dr. Maria Jose Binetti

Instituto Interdisciplinario de Estudios de Género Facultad de Filosofía

Letras Consejo Nacional de Investigaciones Científicas

Técnicas University of Buenos Aires,

Puan 480, Buenos Aires,

Argentina

mjbinetti@gmail.com

Assoc. Prof. PaedDr. Miroslav Tvrdoň, PhD.

Constantine the Philosopher University in Nitra,

94901 Nitra

Slovakia

mtvrdon@ukf.sk

Assoc. prof. Kristina Klasnja, PhD.

Peoples' Friendship University (RUDN University)

Miklukho-Maklaya street, 6

Russia

akhnina-kv@rudn.ru

Assoc. prof. Tatiana A. Baklashova, PhD

Institute of Psychology and EducationKazan (Volga region) Federal University 18

Kremlyovskaya Str.420008 Kazan

Russia

ptatyana2011@mail.ru 
doc. PhDr. PaedDr. Slávka Krásna, PhD., Ph.D.

DTI University

Dubnica nad Váhom

Slovakia

krasna@dti.sk

ORCID ID: https://orcid.org 0000-0001-5287-682X 